\title{
Predicting and Enhancing the Ion Selectivity in Multi-ion Capacitive Deionization
}

Johan Nordstrand and Joydeep Dutta*

Functional Materials, Applied Physics Department, SCI School, KTH Royal Institute of

Technology, Isafjordsgatan 22, SE-16440 Kista Stockholm, Sweden

\section{Supporting information}

S-1 Program files for the calculating ion removal

S-2 Derivation of batch-mode equilibrium removal

S-3 Estimation of removal for the sample from New Delhi

\section{Total number of items}

Pages: 6

Figures: 0

Tables: 1

Equations: 3 


\section{S-1 PROGRAM FILES}

\section{S-2. EQUILIBRIUM OR INITIAL CONCENTRATION}

In the main text, it was shown that the relative adsorption between ionic species in a multi-ion solution is proportional to the relative equilibrium concentration. However, expressing the equation in terms of the initial concentration would be preferable since it is relatively easy to extract. If a continuous-mode operation is performed, the equilibrium concentration will be the same as the initial concentration. In a batch mode, the equilibrium concentration can still be exchanged for the initial concentration if the adsorption strength is similar between the species $(\alpha \approx 1)$, or if the removal is small (Equation $\mathrm{S} 1$ ).

$$
\begin{aligned}
& \frac{c_{a d s}^{(i)}}{c_{a d s}^{(j)}}=\alpha \frac{c_{e}^{(i)}}{c_{e}^{(j)}}=\alpha \frac{c_{0}^{(i)}-c_{a d s}^{(i)}}{c_{0}^{(j)}-c_{a d s}^{(j)}} \\
& \rightarrow \frac{c_{0}^{(j)}-c_{a d s}^{(j)}}{c_{a d s}^{(j)}}=\alpha \frac{c_{0}^{(i)}-c_{a d s}^{(i)}}{c_{a d s}^{(i)}} \\
& \rightarrow \frac{c_{0}^{(j)}}{c_{a d s}^{(j)}}-1=\alpha\left(\frac{c_{0}^{(i)}}{c_{a d s}^{(i)}}-1\right) \\
& \rightarrow \frac{c_{0}^{(j)}}{c_{a d s}^{(j)}}=\alpha \frac{c_{0}^{(i)}}{c_{a d s}^{(i)}}+(1-\alpha) \\
& \rightarrow \frac{c_{a d s}^{(i)}}{c_{a d s}^{(j)}}=\alpha \frac{c_{0}^{(i)}}{c_{0}^{(j)}}+(1-\alpha) \frac{c_{a d s}^{(i)}}{c_{0}^{(j)}}
\end{aligned}
$$




\section{S-3. ESTIMATED REMOVAL FOR GROUNDWATER SAMPLES FROM NEW DELHI}

(INDIA)

In the example in the main text, a sample based on water from the Delhi region was investigated. The details of the calculation are presented here.

While the study contained many different water samples, the typical values for the mass concentrations of the eight most concentrated species, and fluoride, are shown in Table S1. The study did not use capacitive deionization, meaning that $\alpha$ and $\kappa$ values cannot be extracted from that paper. For clarity, we will, therefore, assume that all monovalent ions share $\alpha$ and $\kappa$ values and that all divalent ions similarly share values. Specifically, the values from the calibration in the 'Implementation' section will be used; that is, monovalent ions have $\alpha=1$ and $\kappa=1$, while the divalent ions have $\alpha=7.2$ and $\kappa=1.1$.

For a batch-mode experiment, the concentrations at equilibrium for ions of the same charge sign can be calculated at equilibrium based on Equation S2. The same equation holds for short cycles if $\alpha$ is exchanged for $\kappa$. We will assume that fluoride is the most critical ion species to remove and that its concentration should be reduced from $3 \mathrm{mg} / 1$ to $1.5 \mathrm{mg} / 1$ (so $c_{a d s}^{(b)} / c_{e}^{(b)}=$ 1.5/1.5 = 1). Inserting this, the $\alpha$ values, and the initial concentrations into Equation S2 means that the removal $c_{a d s}^{(i)}$ of all anions can be calculated. Note that the concentration in Equation $\mathrm{S} 2$ is in units of $[\mathrm{mmol} / \mathrm{l}]$; that is, $c_{a d s}^{(i)}[\mathrm{mmol} / \mathrm{l}]=c_{a d s}^{(i)}[\mathrm{mg} / \mathrm{l}] / M[\mathrm{~g} / \mathrm{mol}]$, where $\mathrm{M}$ is the molar mass. 


$$
\frac{c_{a d s}^{(i)}}{c_{0}^{(i)}-c_{a d s}^{(i)}}=\alpha \frac{c_{a d s}^{(b)}}{c_{e}^{(b)}}
$$

To calculate the removal of cations, note that charge neutrality requires the net charge removal to be zero. Thus, Equation S2 can be taken to relate the different cations adsorption to each other, while Equation S3 determines the total cation adsorption. Together, these form a system of equations that can be computed numerically.

$$
\Sigma_{i} c_{a d s}^{(i)} z=0
$$

By solving the presented system of equations, the removal of all cations can thus be calculated in addition to the removal of anions. Software that automates this process and solves the equations presented in this example is presented in Supplementary S-0. 


\begin{tabular}{|l|l|l|l|l|l|}
\hline Name & $\mathbf{m g} / \mathbf{L}$ & charge & g/mol & $\boldsymbol{\alpha}$ & $\boldsymbol{\kappa}$ \\
\hline $\mathrm{Na}$ & 247 & 1 & 23 & 1 & 1 \\
\hline $\mathrm{K}$ & 29 & 1 & 39 & 1 & 1 \\
\hline $\mathrm{Ca}$ & 109 & 2 & 40 & 7.2 & 1.1 \\
\hline $\mathrm{Mg}$ & 63 & 2 & 24 & 7.2 & 1.1 \\
\hline $\mathrm{HCO} 3$ & 419 & -1 & 61 & 1 & 1 \\
\hline $\mathrm{Cl}$ & 419 & -1 & 35 & 1 & 1 \\
\hline $\mathrm{SO} 4$ & 124 & -2 & 96 & 7.2 & 1.1 \\
\hline $\mathrm{NO} 3$ & 31 & -1 & 62 & 1 & 1 \\
\hline F & 3 & -1 & 18 & 1 & 1 \\
\hline
\end{tabular}

Table S1: The mass concentration, charge and molar mass of the eight most concentrated ionic species and fluoride from ref. ${ }^{52}$. The $\alpha$ and $\kappa$ values were assumed to be equal between the different monovalent or divalent ions. Their values were based on the calibration in the 'Implementation' section. 